\title{
CIRNAS: COLLABORATIVE IMMUNE REAGENT NETWORK FOR AQUACULTURED SPECIES
}

\author{
${ }^{1}$ Irene Salinas, ${ }^{2}$ Oriol J. Sunyer, ${ }^{3}$ John D. Hansen \\ ${ }^{1}$ Center for Evolutionary and Theoretical Immunology, Department of Biology, University of New \\ Mexico, Albuquerque, New Mexico 87131, USA \\ ${ }^{2}$ Department of Pathobiology, School of Veterinary Medicine, University of Pennsylvania, \\ Philadelphia, PA, USA. \\ ${ }^{3}$ U.S. Geological Survey, Western Fisheries Research Center, Seattle, WA, USA.
}

Cultured fish represent a critical food resource and efficient means for providing protein for human consumption. Nevertheless, disease limits the sustainability and production of this industry. A major factor restricting the advancement of basic and applied research for fish health is the lack of immunological reagents. These reagents are required for evaluating shifts in immunity during infection and vaccination against pathogens that limit the full potential of aquaculture. CIRNAS (http://biology.unm.edu/CIRNAS) is a collaborative network with a goal of serving the aquaculture community by advancing the availability of immunological resources and knowledge base for fish health. Four fish species: Atlantic salmon, rainbow trout, channel catfish and Nile tilapia will be targeted. Antibody panels and immune assays will be developed to assess the contribution of different leukocyte subsets and effector molecules that cannot be currently measured during disease and vaccination due to lack of corresponding reagents. Specific focus areas include the development of reagents for mucosal immunity and vaccinology. Importantly, a major objective of CIRNAS is to establish an international, collaborative network for improving health, safety and production of aquacultured fish by partnering with other fish health researchers. By doing so, the project will advance the development of collaborative research projects worldwide for diseases impacting production and fish health. Current and proposed research will be presented.

Key Words: trout, salmon, tilapia, catfish, reagent

${ }^{\S}$ Corresponding author: Tel.: 001.206.526.6588; Fax: 001.206.526.6654

E-mail address: John D. Hansen, jhansen @usgs.gov 